\title{
Electrocatalytic Reaction Driven Flow: Role of pH in Flow Reversal
}

\author{
Abimbola A. Ashaju, Veerle Otten, Jeffery A. Wood, and Rob G. H. Lammertink*
}

Cite This: J. Phys. Chem. C 2021, 125, 24876-24886

Read Online

ABSTRACT: Immobilized bimetallic structures generate fluid flow during electrocatalytic reactions with hydrogen peroxide, which is typically driven from the anodic metal to the cathodic metal similar to an electroosmotic flow. However, under low reactive regimes, the generated flow becomes fully reversed, which cannot be explained by the classical electroosmotic theory. This work aims at unraveling the origin and dynamics of this flow hysteresis through a combined experimental and numerical approach. The key electrocatalytic parameters that contribute to flow reversal are analyzed experimentally and numerically under low reactive regimes induced by bulk $\mathrm{pH}$ variations. The proton gradient that initiates chemomechanical actuation is probed with the use of fluorescence lifetime imaging. The fluid flow dynamics

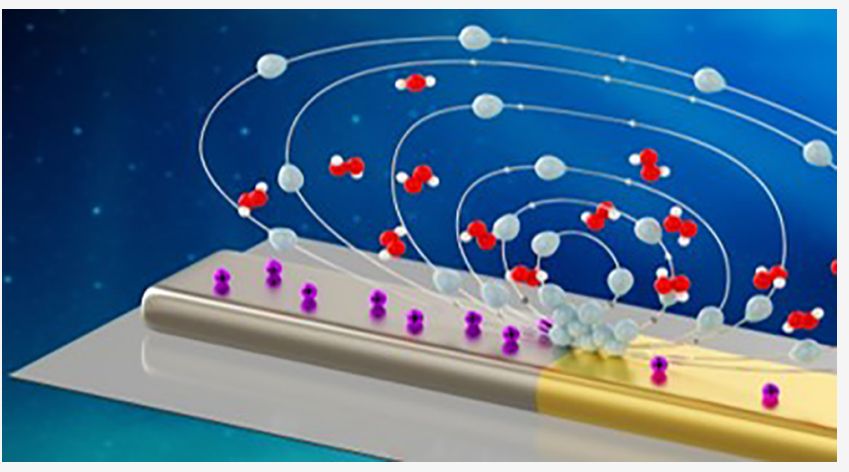
under reactive regimes are visualized by the use of particle tracking. Our numerical simulations elucidate the role of $\mathrm{pH}$ variations and additional ionic species (counterions) toward flow reversal. The combination of these techniques highlights the interplay between electrocatalytic and electrokinetic phenomena on the occurrence of flow reversal.

\section{INTRODUCTION}

Artificial micromotors are devices capable of converting energy to forces and movements. They were engineered by researchers from multiple backgrounds to replicate the complex functionalities of biological motors inspired by nature. ${ }^{1-3}$ A good example concerns the autonomous motion of catalytic nanorods within a fluid medium that functions primarily on chemical energy conversion harvested from the surrounding aqueous fluid., movement of the flagellum, bacteria, and other biological motors that are powered by chemical energy sourced from adenosine triphosphate (ATP) for vital functions in living organisms. The capabilities of micromotors have been extended through the immobilization of the nanorod in the form of a micropump to fluid flow driven by electrochemical gradients obtained from electrocatalytic reactions. ${ }^{6-8}$

Electrocatalytic reaction driven flow (ERDF) is driven by a self-induced electric field that is generated through a gradient of ionic intermediates and powered by the decomposition of a fuel catalyzed by a bimetallic pair. A typical bimetallic system consists of platinum and gold electrodes, immobilized on a substrate, that catalyze the electrochemical decomposition of hydrogen peroxide through a series of redox half-reactions ((1)

$$
\mathrm{H}_{2} \mathrm{O}_{2} \rightarrow 2 \mathrm{H}^{+}+\mathrm{O}_{2}+2 \mathrm{e}^{-}
$$

$$
\mathrm{H}_{2} \mathrm{O}_{2}+2 \mathrm{H}^{+}+2 \mathrm{e}^{-} \rightarrow \mathrm{H}_{2} \mathrm{O}
$$

and (2)). The decomposition of hydrogen peroxide by platinum can proceed via different pathways. ${ }^{9}$ During oxidation reaction at the $\mathrm{Pt}$ anode, oxygen is produced, while protons and electrons are generated. Simultaneously, the electrons are transferred to and protons are consumed at the Au cathode via the reduction reaction. The proton flux evolves into a concentration gradient, which creates an electrical body force on the charged near-electrode liquid layer, which drives fluid flow (see Figure 1).

Besides the above electrode reaction, bulk dissociation of hydrogen peroxide will generate $\mathrm{HO}_{2}{ }^{-}$along with $\mathrm{H}^{+}$ions. ${ }^{10,11}$ This can provide other ions to carry the ionic current, affecting the diffusion potential. Here, we consider the simplest reaction form that yields protons, combined with bulk water dissociation only.

ERDF has been explored in various instances, such as the enhancement of mass transport across a membrane in a compartment-less fuel cell, ${ }^{12}$ oxygen generation, ${ }^{13}$ and colloidal crystallization. ${ }^{14,15}$ The reaction mechanism involving proton generation and consumption, as well as its impact on the reaction kinetics and the resulting fluid flow, is not fully understood. The interplay between these coupled phenomena

Received: July 20, 2021

Revised: October 13, 2021

Published: November 5, 2021

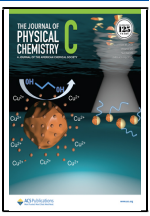




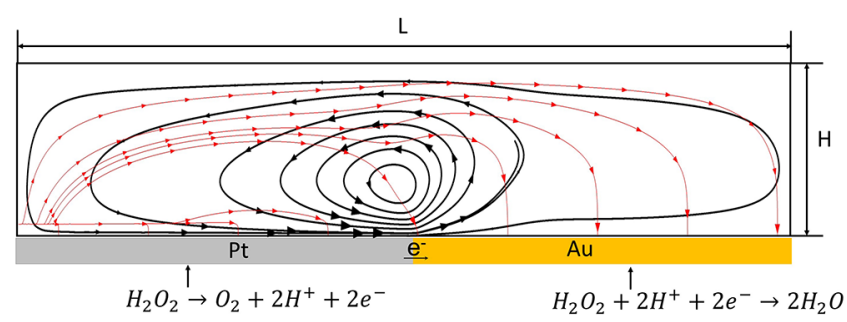

Figure 1. Schematic of $\mathrm{Pt}-\mathrm{Au}$ bimetallic system that catalyzes the decomposition of hydrogen peroxide yielding ionic species which generate an electric field (red streamlines) that drives fluid flow (black streamlines). $L=10 \mathrm{~mm}$ is the length of bielectrode, and $H$ is the height of the cell, where $H=1500 \mu \mathrm{m}$ for the FLIM setup and $H=$ $500 \mu \mathrm{m}$ for the fluid flow setup.

under different reaction regimes is the main focus of this paper. Many studies have focused on the chemomechanical mechanism that drives ERDF, and they will be briefly highlighted. The fuel that powers chemical energy conversion has been a subject of interest in some studies. Hydrogen peroxide decomposition has been central to many proof-ofconcept studies; however, other fuel substitutes such as hydrazine derivatives and iodine have found relevance in generating ERDF, which eliminates the complexities of oxygen bubbles associated with peroxide decomposition. ${ }^{8,16}$ The reactivity of the bimetallic metals is central to an electrochemical conversion that generates ERDF, and highly reactive metallic pairs that promote electrocatalytic conversion have been researched as suitable alternatives to the classical $\mathrm{Pt}-\mathrm{Au}$ bimetallic pair. $^{17}$

The electrochemical conversion has been characterized using direct current-potential measurements ${ }^{6}$ and TAFEL analysis, ${ }^{17,18}$ where the potential induced during the electrochemical reaction and the catalytic current that is associated with the reaction kinetics are measured. The resulting fluid flow concerned an electroosmotic flow pattern where the flow is driven from the anode to the cathode and recirculates within a confined system. ${ }^{6,7}$ The velocity magnitude, albeit highly sensitive to $\mathrm{pH}$ variations, can be tuned by the fuel concentration. ${ }^{19}$

Flow reversal occurs whenever the induced fluid flow that is typically driven from the anode to the cathode becomes redirected in the sense opposite to an electroosmotic flow. According to the Helmholtz-Smoluchowski equation, the zeta $(\zeta)$ potential is an important parameter that contributes to electroosmotic flow velocity $\mathbf{u}$, and its sign relative to the electric field direction dictates the flow direction. Subramanian and Catchmark experimentally demonstrated flow reversal by reengineering the $\zeta$ potentials of the bimetallic electrode. ${ }^{20}$ They coated the electrode surface with self-assembled monolayers that modify the surface charge and the resulting $\zeta$ potential sign. For the case where the $\zeta$ potential of the electrode was made positive, the flow becomes reversed. The Helmholtz-Smoluchowski equation for flow is given as $\mathbf{u}=-\frac{\varepsilon}{\eta} \zeta \mathbf{E}$, where $\varepsilon$ is the permittivity of the fluid and $\mathbf{E}$ is the electric field.

Aside from the flow reversal governed by the $\zeta$ potential switch, flow reversal can be induced by the electric field reversal that stems from the electrocatalytic reaction. Ibele et $\mathrm{al}^{8}$ reported reversal of flow based on the fuel choice that impacts the induced potential and tangential electric field. Two variants of hydrazine were used for this purpose, $\mathrm{N}_{2} \mathrm{H}_{4}$ and
$\mathrm{N}_{2} \mathrm{Me}_{2} \mathrm{H}_{2}$, with a Au-Pd bimetallic system. The electrodes exhibited different roles for these fuel variants as evidenced by their mixed potentials, where $\mathrm{Au}$ having a higher mixed potential $(-310 \mathrm{mV})$ for $\mathrm{N}_{2} \mathrm{H}_{4}$ assumes the cathodic role and flow is driven toward it. In the case where $\mathrm{N}_{2} \mathrm{Me}_{2} \mathrm{H}_{2}$ was used, the role of the electrodes became reversed and $\mathrm{Pd}$ having a higher mixed potential $(-70 \mathrm{mV})$ acted as the cathode with flow being driven from Au to Pd. Although they described the flow direction as being governed by the resultant effects of the electrode $\zeta$ potential in conjunction with the $\zeta$ potentials of the colloidal particles, the different mixed potentials exhibited by the electrodes in different solutions would create variations in the bimetallic potential, which has consequences for the electric field and strengthens the argument for a flow reversal that is driven by the electrocatalytic reaction.

In an earlier paper, we reported the observation of flow reversal under low anodic reactive regimes $\left(D a_{\mathrm{a}}<100\right) .{ }^{19}$ The anodic Damköhler number, $D a_{a}$, defines the rate at which oxidation reaction proceeds that leads to the generation of protons. The slow oxidation reaction becomes the overall limiting mechanism, and the proton gradient across the bielectrode is diminished. The proton gradient serves as the basis for the actuation of the bimetallic system, and its weakened form has negative consequences for the induced electric field and body forces that drive the fluid flow. However, the dynamics of the proton gradient under reactive regimes and the effects on the fluid flow are yet to be fully understood. It is the aim of this study to investigate the interplay between these two main phenomena.

Fluorescence imaging has emerged as a useful tool for probing electrode-electrolyte interfacial processes ${ }^{21-24}$ as well as ion transport in systems. ${ }^{25,26}$ This technique involves the use of an ionic chemical species that modulates directly or indirectly a fluorescence signal that emanates from a reaction mechanism. The modulation could be a change in quantum yield or emission wavelength, which directly affects the amplitude of the signal. ${ }^{23}$ Farniya et al. ${ }^{18}$ employed fluorescence intensity based on ratiometric measurements to track the proton gradient during the catalytic reaction involving $\mathrm{Pt}-\mathrm{Au}$ in $\mathrm{H}_{2} \mathrm{O}_{2}$ They showed that the proton concentration changes by almost an order of magnitude along the radial direction of the bielectrode. However, this technique has its limitation as the fluorophores have the tendency to photobleach, which affects the accuracy of the result. Fluorescence lifetime imaging (FLIM), on the other hand, is based on the average decay time of a fluorophore's excited state, depending on its interactions with ions and dynamics in the system. ${ }^{27}$ FLIM offers key advantages over fluorescent intensity in terms of insensitivity to the fluorescent dye concentration and excitation intensity, ${ }^{28}$ and spatial-temporal resolution.

Given the advantages provided by FLIM, the reactioninduced proton gradient at the bielectrode/electrolyte interface was characterized using this technique. Furthermore, a twodimensional model based on the Poisson-Nernst-Planck and Stokes equations was used to elucidate the role of the proton gradient on the induced electric field and other key elements that control the resulting mass transport characteristics. The reaction regimes that concern ERDF and reversed flow are fully analyzed. A complete understanding of the reaction driven fluid flow opens more opportunities for fluid control and exploitation in targeted systems. 


\section{EXPERIMENTAL DETAILS}

Platinum and gold bielectrodes were fabricated on planar glass substrates via photolithography, metal sputtering, and liftoff. The glass substrates were spin-coated with positive photoresist, followed by exposure to UV light through a photomask and development. Tantalum was presputtered on the substrates to improve adhesion, before platinum and gold metals were deposited through DC sputtering (MESA+ NanoLab clean room in-house equipment, TCOathy). The photoresist negative pattern was removed via a liftoff process in acetone. Two design variants were fabricated: $\mathrm{Pt}-\mathrm{Au}$ bimetallic and interdigitated electrodes (Figure 2). The bimetallic electrode

(a)

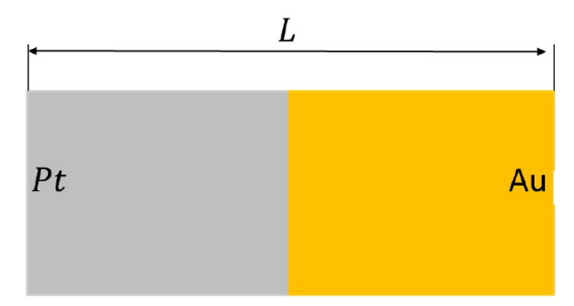

(b)

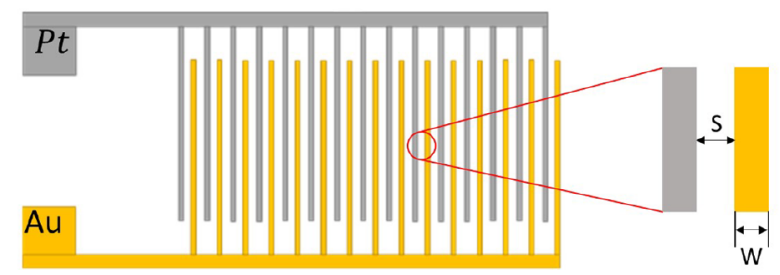

Figure 2. Schematic diagrams showing $\mathrm{Pt}-\mathrm{Au}$ (a) bimetallic electrode with total length $L=10 \mathrm{~mm}$ and (b) interdigitated electrodes with interspacing $S=40 \mu \mathrm{m}$, electrode width $W=50 \mu \mathrm{m}$, and total surface area $A=9.08 \times 10^{-6} \mathrm{~m}^{2}$.

consists of a $\mathrm{Pt}-\mathrm{Au}$ galvanic pair that maintains direct contact and is used for the microscopic observations. The interdigitated electrodes consist of electrode fingers of alternating platinum and gold that are connected to separate terminals and is designed primarily for electrochemical characterization. Before any experimental measurements, the surface was cleaned by rinsing with isopropyl alcohol and DI water, followed by oxygen plasma treatment (plasma power $=100 \mathrm{~W}$, oxygen gas pressure $=0.5 \mathrm{bar}, 5 \mathrm{~min}$ ).

2.1. Electrochemical Measurement. The interdigitated electrodes were bonded to a printed circuit board that allows external connection to an eletrochemical workstation as well as external control of the electrode potential. Electrochemical measurement was performed by using an Autolab PGSTAT204 potentiostat, running NOVA 2.0 software, with a three-electrode cell comprising the platinum and gold interdigitated electrodes with a micro $\mathrm{Ag} / \mathrm{AgCl}$ reference electrode (eDAQ model ET073) in $2 \mathrm{~mol} / \mathrm{m}^{3} \mathrm{H}_{2} \mathrm{O}_{2}$. Either the $\mathrm{Pt}$ or the $\mathrm{Au}$ electrode was subjected to potential sweeps with the oxidation and reduction range with a scan rate of 1 $\mathrm{mV} / \mathrm{s}$, and the resulting current response was recorded.

2.2. Fluid Flow Visualization. To visualize the induced flow patterns, we introduced fluorescent particles (carboxylated-modified FluoSpheres), $1 \mu \mathrm{m}$ in diameter, in hydrogen peroxide solution (Sigma-Aldrich), which was diluted in MilliQ water $(\rho>18 \mathrm{M} \Omega \mathrm{cm})$. The $\mathrm{pH}$ of the resulting mixture was adjusted with $\mathrm{HCl}$ or $\mathrm{NaOH}$. The conductivities of the prepared solutions were measured with a conductivity probe (WTW Cond 3110, Weilheim, Germany) to be in the range $10-30 \mu \mathrm{S} / \mathrm{cm}$. A fluidic chamber with height $H=500 \mu \mathrm{m}$ was constructed around the bimetallic electrode using poly(methyl methacrylate) (PMMA) glass and covered at the side with a glass coverslip (see Figure 3a). The particle and hydrogen peroxide solution was introduced into a chamber of height $H=$ $500 \mu \mathrm{m}$. The particle movement that was driven by the fluid flow was observed from the horizontal plane with an optical microscope (Carl Zeiss Axio Observer Z1, 20X), while images were acquired at a frame rate of $10 \mathrm{fps}$. Image processing was conducted with ImageJ, and the particle displacement vectors were obtained with the open source tool PIVlab. ${ }^{29}$

2.3. Fluorescence Lifetime Imaging Microscopy. The proton concentrations close to the surface of the bielectrode were determined with fluorescence lifetime imaging microscopy (FLIM; LIFA, Lambert Instruments, The Netherlands). The FLIM setup consists of an image intensifier (TRiCAM), frequency generator, and LED light source $(3 \mathrm{~W}$, emission wavelength of $460 \mathrm{~nm}$ ), which are connected to a Zeiss Axio Vert A1 inverted microscope (Zeiss, Germany) that is equipped with a filter cube (443-493 $\mathrm{nm}$ band-pass excitation, 500-650 nm band-pass emission). Data acquisition and (a)

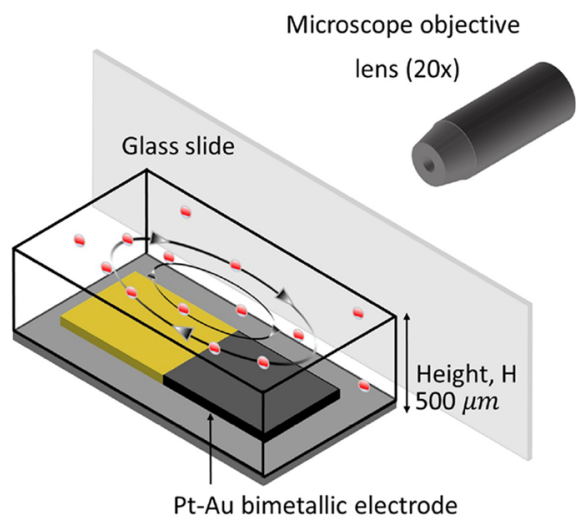

(b)

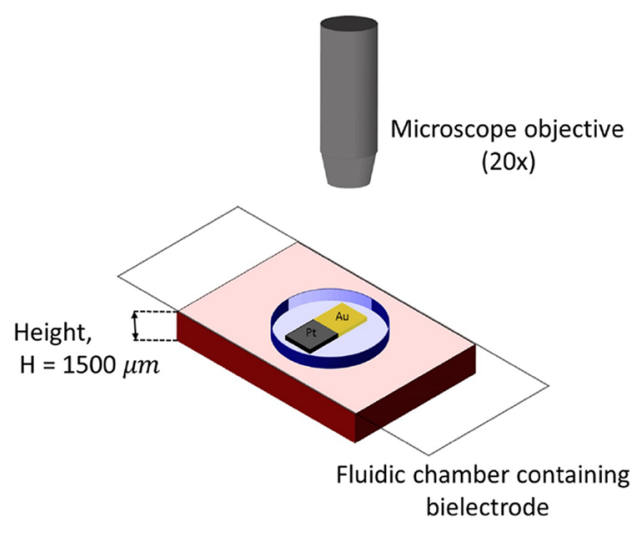

Figure 3. Schematic diagrams of experimental setup for (a) fluid flow study and (b) fluorescence lifetime imaging. Electrode is enclosed within the chamber and filled with tracer particle and $\mathrm{H}_{2} \mathrm{O}_{2}$ solution for flow visualization and fluorescent dye with $\mathrm{H}_{2} \mathrm{O}_{2}$ solution for proton gradient imaging. 
processing were performed under the frequency domain mode using LI-FLIM software. A homodyne detection method was used in extracting the phase shift and modulation depth data from the acquired fluorescence signal, by modulating the light source and the intensified camera at a modulation frequency of $40 \mathrm{MHz}$ and a phase angle of $250^{\circ}$. The modulation frequency $f$ used in measurements is the optimal frequency at which small changes of the lifetime for the sample being studied can generate large response in terms of the phase shift and modulation depth and was taken as $\frac{1}{2 \pi \tau}$, where $\tau$ is the fluorescent lifetime of interest. For every data acquisition, 12 images were obtained from different phase offsets, while the phase shift and modulation depth that yields the lifetime for each pixel were determined by fitting the fluorescence signal with a sine function. For the FLIM experiments, hydrogen peroxide solutions containing the LysoSensor $\mathrm{pH}$ probe (LysoSensor green DND-189, ThermoFisher scientific) were used. The LysoSensor $\mathrm{pH}$ probe can fluoresce within an acidic to near-neutral medium (indicative of $\mathrm{pH}$ range 5-6). The hydrogen peroxide and fluorescent dye solution was introduced into an imaging chamber (height $H=1500 \mu \mathrm{m}$ ) that contains the bimetallic electrode and is covered with a glass slide (Figure 3b). When a clear focus of electrodes was obtained, the height at this point was set as the origin $(H=0$ $\mu \mathrm{m})$ and image frames of the fluorescence lifetime were acquired from the top view. Before the FLIM measurements, calibration between the $\mathrm{pH}$ and the fluorescence lifetime of the LysoSensor probe was performed. Samples consisting of the LysoSensor probe and $\mathrm{pH}$ buffers (phosphate buffer, potassium hydrogen phthalate buffer, Thermo Fisher Scientific) were prepared within the $\mathrm{pH}$ range $4-8$, while the $\mathrm{pH}$ was adjusted accordingly by $\mathrm{HCl}$ or $\mathrm{NaOH}$.

The calibration curve for the fluorescence lifetime experiments was obtained by fitting the plot of the fluorescence lifetime vs proton $\left(\mathrm{H}^{+}\right)$concentration using a Boltzmann sigmoid equation (see Figure 4 ):

$$
\tau(\mathrm{ns})=A_{2}+\frac{A_{1}-A_{2}}{1+\mathrm{e}^{\left(\mathrm{pH}-\mathrm{p} K_{\mathrm{a}}\right) / \mathrm{d} x}}
$$

where $A_{1}$ and $A_{2}$ are the upper and lower limits of the fluorescence lifetime, respectively, and $\mathrm{d} x$ is the slope at the inflection point. The calibration curve demonstrates a steep transition and a high sensitivity from $\mathrm{pH} 5.4$ to 6 due to the quantum yield of the LysoSensor probe during protonation. The protonation process enhances the fluorescence response of the dye molecule by its weakly basic chain that results in an increase in fluorescent intensity and lifetime signals at lower $\mathrm{pH}^{30}$ The Boltzmann sigmoid fit yielded an apparent $\mathrm{p} K_{\mathrm{a}}$ of 5.8 , comparable to what was reported in other works ${ }^{30,31}$

\section{THEORY AND NUMERICAL SIMULATION}

Following our previous work, we used a $2 \mathrm{D}$ numerical model that is based on the dimensionless Poisson-Nernst-Planck equations for the transport of ionic species, coupled with the Stokes equation for fluid flow (see ref 19). The model was developed according to the dimensions of the experimental setup. For the fluid flow analysis (side view) the channel height is $H=500 \mu \mathrm{m}$ (Figure 3a) with an aspect ratio $H / L=0.05$. For the FLIM experimental study, the channel height is $H=$ $1500 \mu \mathrm{m}$ (Figure $3 \mathrm{~b}$ ). The width $L$ is $10 \mathrm{~mm}$ for both cases. The numerical models were always solved for the $z-x$ plane, representing the side view.

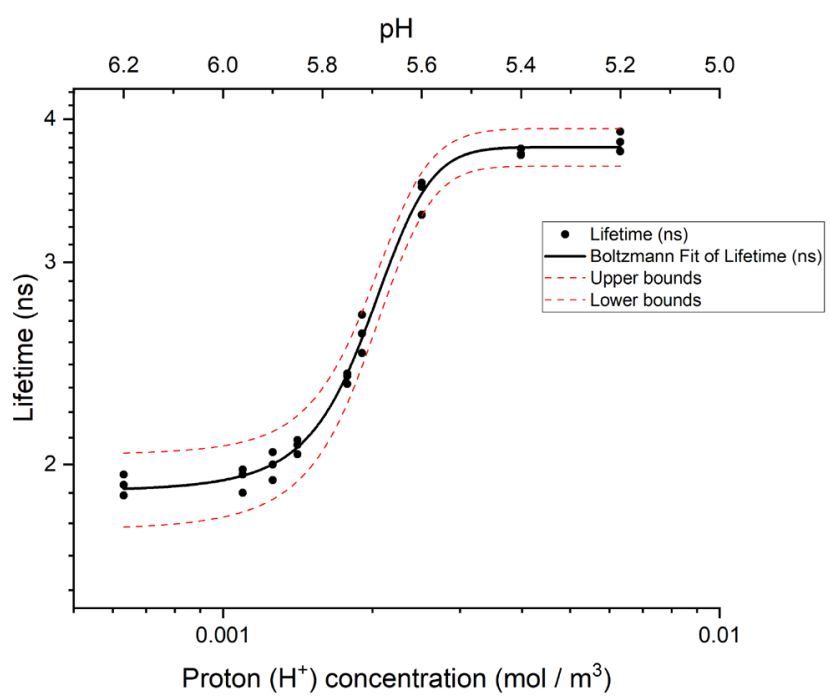

Figure 4. Lifetime vs proton concentration and calibration curve for FLIM measurements on semilog scale. Data was plotted within the $\mathrm{pH}$ range 5-6, where the fluorescence dye is sensitive to protonation. Solid line corresponds to the best fit to a Boltzmann sigmoid model, and the dashed lines refer to the $95 \%$ confidence interval.

3.1. Governing Equations. The concentrations of ionic species are coupled with the potential $\phi$ through Poisson's equation (eq 4) to resolve the potential distribution. The

$$
-2 \epsilon^{2} \nabla^{2} \phi=\Sigma_{i} z_{i} c_{i}
$$

induced potential, which is a dimensionless quantity, is obtained by normalizing the electrostatic potential $\tilde{\phi}$ by the thermal voltage $V_{T}=\frac{R T}{F} \approx 25 \mathrm{mV}$, where $T$ is the temperature, $F$ is the Faraday constant $\left(F=96485 \mathrm{C} \mathrm{mol}^{-1}\right)$, and $R$ is the universal gas constant $(8.314 \mathrm{~J} / \mathrm{K} \cdot \mathrm{mol})$. The tilde symbol $(\sim)$ represents a dimensional quantity.

At the interface between the bielectrode and the electrolyte solution, a double layer is formed that is described by the dimensionless Debye length $\epsilon$, obtained by normalizing the Debye length $L_{\mathrm{D}}=\sqrt{\frac{\varepsilon V_{T}}{2 F \tau_{\infty}}}$ with the length of the bielectrode $\epsilon=\frac{L_{\mathrm{D}}}{L}$. The thickness of the Debye length $L_{\mathrm{D}}$ for the electrolyte at $\mathrm{pH} 6$ is $304 \mathrm{~nm}$.

The transport of ionic species is accounted for by the Nernst-Planck equation (eq 5) by solving the advection, diffusion, and electromigration contributions involving the 1:1 electrolyte.

$$
\frac{\partial c_{i}}{\partial_{t}}+\nabla \cdot\left(\mathbf{u} c_{i}\right)=D_{i} \nabla \cdot\left(\nabla c_{i}+z_{i} c_{i} \nabla \phi\right)+R_{i}
$$

where $\mathbf{u}$ is the velocity magnitude and $c_{i}=\frac{\tilde{c}_{i}}{\tilde{c}_{\mathrm{w}}}$ is the concentration of species $i$ normalized by the bulk concentrations of $\mathrm{H}^{+}$and $\mathrm{OH}^{-}$in pure water at neutral $\mathrm{pH}\left(\tilde{c}_{\mathrm{w}}=1 \times\right.$ $\left.10^{-7} \mathrm{M}\right)$, whose diffusion coefficient and valence are $D_{i}=\frac{\tilde{D}_{i}}{\tilde{D}_{0}}$ and $z_{i}$ respectively. $\tilde{D}_{0}$ is the diffusivity of hydroxide ions $\left(\tilde{D}_{0}=\right.$ $5.27 \times 10^{-9} \mathrm{~m}^{2} / \mathrm{s}$ ) at $T=298 \mathrm{~K}$ selected as a reference value. The self-ionization of water is included as a bulk reaction $R_{i}$, defined as 


$$
R_{i}=D a_{\mathrm{b}}\left(\frac{K_{\mathrm{w}}}{\tilde{c}_{\infty}^{2}}-c_{\mathrm{H}^{+} \mathcal{C}_{\mathrm{OH}^{-}}}\right)
$$

where $K_{\mathrm{w}}$ is the water ionization constant expressed as $K_{\mathrm{w}}=$ $\left[\mathrm{H}^{+}\right]\left[\mathrm{OH}^{-}\right]=10^{-14} \mathrm{M}^{2} . D a_{\mathrm{b}}$ is the bulk Damköhler number defined as $D a_{\mathrm{b}}=\frac{k_{\mathrm{rb}} L^{2} \tilde{c}_{\infty}}{\tilde{D}_{0}}, k_{\mathrm{rb}}$ is the water recombination reaction rate constant, and $c_{\mathrm{H}^{+}}$and $c_{\mathrm{OH}^{-}}$are the concentrations of proton and hydroxide species, respectively. In this model, we thus limit ourselves to protons and hydroxide ions and neglect the potential influence of other ions such as bicarbonate from dissolved carbon dioxide and bulk dissociation of hydrogen peroxide.

As we operate at rather low hydrogen peroxide concentrations and at slightly acidic conditions, the contribution from hydroperoxyl ions in the ionic current are neglected. On the basis of a $\mathrm{pK}$ close to 12 , we estimate the hydroperoxyl ion concentration to be about 5 orders of magnitude smaller than the proton concentration. Furthermore, a slower diffusing anion would further enhance the induced diffusion potential and thus the induced velocity. The induced fluid flow is described by the dimensionless Stokes equations (eqs 6 and 7)

$$
\begin{aligned}
& \frac{1}{S_{c}} \frac{\partial \mathbf{u}}{\partial t}=-\nabla p+\nabla^{2} \mathbf{u}-\frac{\kappa}{2 \epsilon^{2}} \rho_{\mathrm{e}} \nabla \phi \\
& \nabla \cdot \mathbf{u}=0
\end{aligned}
$$

under the assumptions of steady-state conditions and low Reynolds number; hence we neglect the nonlinear inertial term. Here, $S c$ is the Schmidt number defined as $S c=\frac{\eta}{\rho D_{0}}$ and of $O\left(10^{3}\right), D_{0}$ is the diffusivity of hydroxide ions as a reference value, and $\kappa=\frac{\varepsilon V_{T}^{2}}{\eta D_{0}}=0.093$ is the electrohydrodynamic coupling constant.

3.2. Boundary Conditions. We impose a no-slip condition, $\mathbf{u}=0$, on all boundaries for Stokes equations. The potential is uniform across the metal surface, as it is a conductor. We further assume a Stern layer model, with a Stern layer described by a constant capacitor. The model resolves the diffuse part of the electric double layer, while the Stern layer is resolved by a linear potential drop across the Stern layer $\left(\Delta \phi_{\mathrm{S}}\right)$ (eq 8) and the potential drop scaled with the ratio between Stern layer thickness $\lambda_{\mathrm{S}}$ and the Debye layer thickness $\lambda_{\mathrm{D}}$ (eq 9).

$$
\begin{aligned}
& \Delta \phi_{\mathrm{S}}=\zeta-\phi_{\text {metal }} \\
& \Delta \phi_{\mathrm{S}} \approx \frac{\lambda_{\mathrm{S}}}{\lambda_{\mathrm{D}}} \zeta
\end{aligned}
$$

Equation 9 assumes a comparable potential gradient in both the Stern layer and the diffuse layer. For the case of an electrolyte having a low salt concentration $\frac{\lambda_{\mathrm{S}}}{\lambda_{\mathrm{D}}} \ll 1$, the potential drop across the Stern layer $\Delta \phi_{\mathrm{S}}$ is extremely small; hence the boundary condition becomes a simple Dirichlet boundary condition $\phi_{\text {metal }} \cong \zeta$, as also described by Moran and Posner. $^{32}$ At the upper boundary of the model $(y=0.15)$, we apply $\frac{\partial \phi}{\partial y}=0$. Sabass and Seifert ${ }^{33}$ considered possible $\mathrm{H}_{2} \mathrm{O}_{2}$ decomposition pathways and affirmed that both anodic and cathodic reactions can be considered linear in hydrogen peroxide as a first-order approach. Following the work of Moran and Posner, ${ }^{32}$ we assumed the electrochemical reaction involving hydrogen peroxide as a single-step process of first order at the anode and the cathodic reaction involving protons as second order. As a result, the reaction kinetics are derived following the Frumkin-Butler-Volmer equation which has been used for studying transport phenomena near electrodes in other works: ${ }^{32,34-36}$

$$
\tilde{j}=k_{\mathrm{a}} \tilde{c}_{\mathrm{R}} \exp \left(\frac{(1-\alpha) n F \Delta \tilde{\phi}_{\mathrm{S}}}{R T}\right)-k_{\mathrm{c}} \tilde{\mathcal{c}}_{\mathrm{O}} \exp \left(\frac{-\alpha n F \Delta \tilde{\phi}_{\mathrm{S}}}{R T}\right)
$$

where $\tilde{j}$ is the proton flux linked to the current density through Faraday's law of electrolysis, $\tilde{j}=i / n F A z ; A$ is the surface area of the electrodes and $n$ is the number of electrons transferred during the reaction. The anodic and cathodic reaction rate constants are denoted as $k_{\mathrm{a}}$ and $k_{\mathrm{c}}$, respectively. Since the potential drop across the Stern layer $\Delta \phi_{\mathrm{S}}$ is extremely small as $\lambda_{\mathrm{S}} / \lambda_{\mathrm{D}} \ll 1$, the exponential term in eq 10 approaches unity, and the Frumkin-Butler-Volmer equation is formulated for the anodic reaction as

$$
\tilde{j}_{\mathrm{Pt}}=k_{\mathrm{a}_{\mathrm{Pt}}} \tilde{c}_{\mathrm{H}_{2} \mathrm{O}_{2}}-k_{\mathrm{c}_{\mathrm{Pt}}} \tilde{c}_{\mathrm{O}_{2}} \tilde{c}_{\mathrm{H}^{+}}{ }^{2}
$$

and for the cathode reaction as

$$
\tilde{j}_{\mathrm{Au}}=k_{\mathrm{a}_{\mathrm{Au}}} \tilde{c}_{\mathrm{H}_{2} \mathrm{O}}{ }^{2}-k_{\mathrm{c}_{\mathrm{Au}}} \tilde{c}_{\mathrm{H}_{2} \mathrm{O}_{2}} \tilde{c}_{\mathrm{H}^{+}}^{2}
$$

The proton current is simplified based on the assumption that the irreversible electrocatalytic reaction for both electrodes proceeds in the forward direction under the Tafel regime, which gives us an oxidation reaction flux $\tilde{j}_{\mathrm{Pt}}$ occurring at the anode as

$$
\tilde{j}_{\mathrm{Pt}}=k_{\mathrm{a}} \tilde{c}_{\mathrm{H}_{2} \mathrm{O}_{2}}
$$

and a reduction reaction flux on the cathode as

$$
\tilde{j}_{\mathrm{Au}}=-k_{\mathrm{c}} \tilde{\mathcal{c}}_{\mathrm{H}_{2} \mathrm{O}_{2}} \tilde{\mathcal{c}}_{\mathrm{H}^{+}}{ }^{2}
$$

The combined electromigration, advective, and diffusive fluxes are matched with the dimensionless form of the rate equations to obtain the proton flux that is applied on individual electrodes. ${ }^{37}$ The respective boundary conditions on platinum and gold become

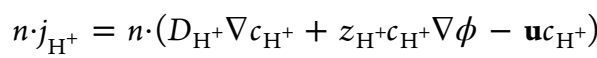

$$
\begin{aligned}
& =\left\{\begin{array}{l}
j_{\mathrm{Pt}}=D_{\mathrm{H}^{+}} D a_{\mathrm{a}} \\
j_{\mathrm{Au}}=-D_{\mathrm{H}^{+}} D a_{\mathrm{c}} c_{\mathrm{H}^{+}}{ }^{2}
\end{array}\right.
\end{aligned}
$$

where $j_{\mathrm{Pt}}$ and $j_{\mathrm{Au}}$ represent the platinum and gold surface proton fluxes, respectively. The oxidation and reduction reactions that occur at $\mathrm{Pt}$ and $\mathrm{Au}$ metals are characterized by the anodic Damköhler number $D a_{\mathrm{a}}=\frac{k_{\mathrm{a}} L \tilde{c}_{\mathrm{H}_{2} \mathrm{O}_{2}}}{\tilde{D}_{+} \tilde{c}_{\infty}}$ and the cathodic Damköhler number $D a_{\mathrm{c}}=\frac{k_{L} L \tilde{c}_{\infty} \tilde{\mathrm{H}}_{\mathrm{H}_{2} \mathrm{O}_{2}}}{\tilde{D}_{+}}$, respectively. The fluxes of anionic species are set to zero at the surface of the bielectrode, giving

$$
n \cdot j_{\mathrm{OH}^{-}}=n \cdot\left(D_{\mathrm{OH}^{-}} \nabla c_{\mathrm{OH}^{-}}+z_{\mathrm{OH}^{-} \mathcal{C}_{\mathrm{OH}^{-}}} \nabla \phi-\mathbf{u} c_{\mathrm{OH}^{-}}\right)=0
$$


(a)

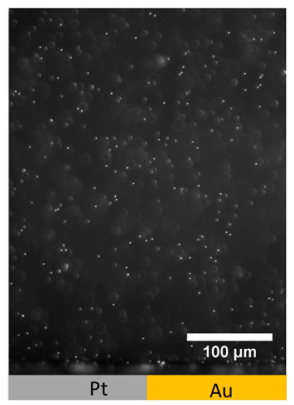

(b)

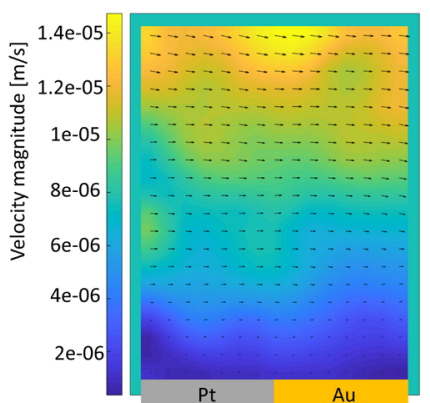

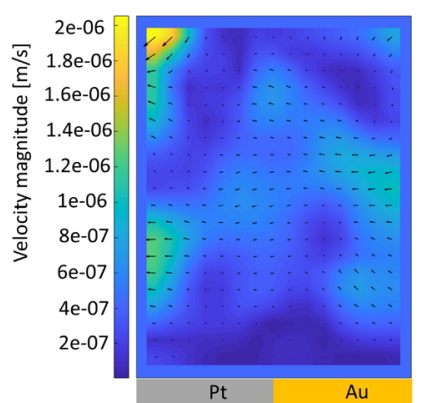

Figure 5. (a) Fluid flow visualization by particle movements in $0.326 \mathrm{M} \mathrm{H}_{2} \mathrm{O}_{2}$. (b) Displacement vectors for fluid flow at bulk pH 5.8. (c) Displacement vectors for the reversed flow at bulk $\mathrm{pH} 4.2$.

(a)

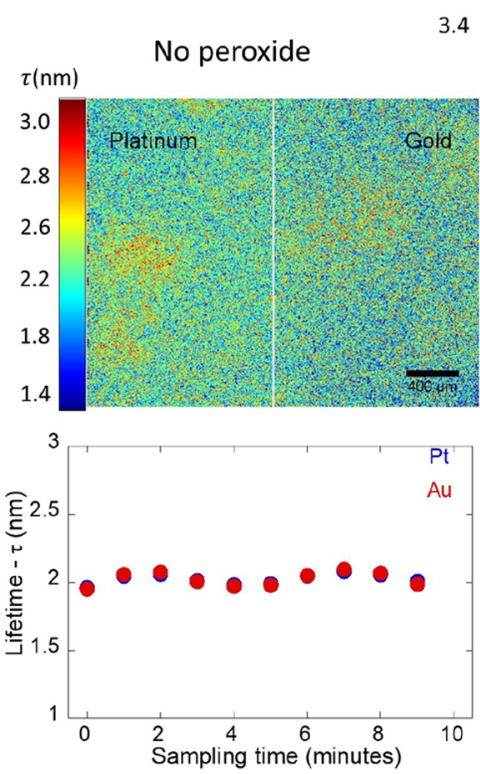

(b)
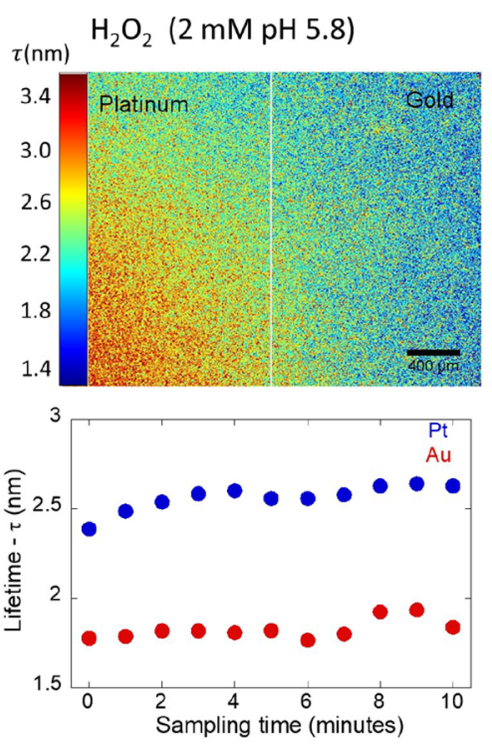

(c)

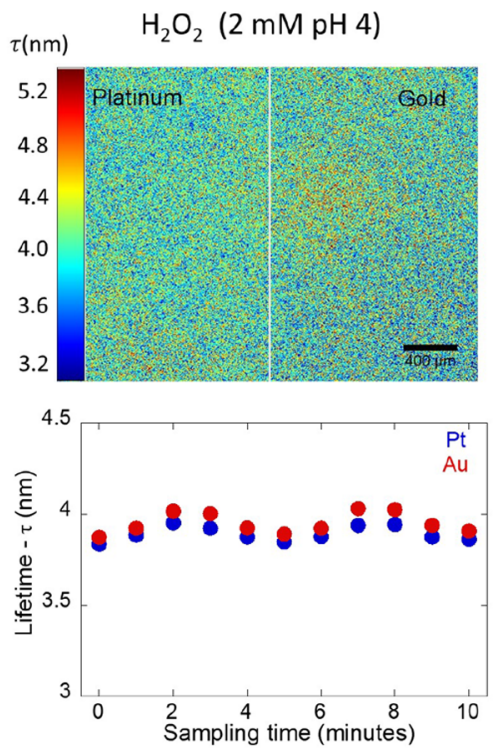

Figure 6. Fluorescence lifetime of $\mathrm{Pt}-\mathrm{Au}$ for (a) no hydrogen peroxide reaction; (b) $2 \mathrm{~mol} / \mathrm{m}^{3} \mathrm{H}_{2} \mathrm{O}_{2}, \mathrm{pH} 5.8$; and $(\mathrm{c}) 2 \mathrm{~mol} / \mathrm{m}^{3} \mathrm{H}_{2} \mathrm{O}_{2}, \mathrm{pH} 4$. Color maps were scaled to fit $98 \%$ of data.

$$
n \cdot j_{\mathrm{Cl}^{-}}=n \cdot\left(D_{\mathrm{Cl}^{-}} \nabla c_{\mathrm{Cl}^{-}}+z_{\mathrm{Cl}^{-}{ }^{-} \mathrm{Cl}^{-}} \nabla \phi-\mathbf{u} c_{\mathrm{Cl}^{-}}\right)=0
$$

At the upper boundary of the model, we set the initial concentration for each ionic species as a Dirichlet condition that is coupled to the bulk electrolyte $\mathrm{pH}$ through $-\log \left(\tilde{\mathcal{c}}_{\infty}\right)$. The $\mathrm{pH}$ of the electrolyte was adjusted during experimental studies with $\mathrm{HCl}$. This was included in the model by using an expression that accounts for the bulk proton concentration due to bulk $\mathrm{pH}$ variations, electroneutrality, and including water self-ionization (eq 18). (The full derivation can be seen in Ashaju et al. ${ }^{19}$ )

$$
\tilde{c}_{\mathrm{H}^{+}}=\frac{\tilde{c}_{\infty}+\sqrt{\left(\tilde{c}_{\infty}\right)^{2}+4 K_{\mathrm{w}}}}{2}
$$

3.3. Simulation Method. Simulations were performed based on the bimetallic current $\left(0.06 \mathrm{~A} / \mathrm{m}^{2}\right)$ that was measured from Tafel plots obtained from electrochemical measurements for the case of $2 \mathrm{~mol} / \mathrm{m}^{3} \mathrm{H}_{2} \mathrm{O}_{2}$ at $\mathrm{pH} 6$ (see Figure S1). Following the approach that was reported elsewhere, ${ }^{19}$ the measured electric current was converted to reaction flux (in mol m $\mathrm{m}^{-2} \mathrm{~s}^{-1}$ ) by using Faraday's law of electrolysis, $j=i / n F A z$, and applied directly to the electrode as a Neumann boundary condition. The effective rate constants for the redox reactions are extracted by using the Frumkincorrected Butler-Volmer equation (eq 10) after computation of the model. The anodic and cathodic rate constants are determined to be $k_{\mathrm{a}}=1.63 \times 10^{-7} \mathrm{~m} / \mathrm{s}$ and $k_{\mathrm{c}}=5.16 \times 10^{-4}$ $\mathrm{m}^{7} \mathrm{~s}^{-1} \mathrm{~mol}^{-2}$, respectively. Both rate constants are used in determining the experimental Damköhler numbers, found to be approximately $\mathrm{Da}_{\mathrm{a}}=350$ and $\mathrm{Da}_{\mathrm{c}}=1$, respectively. Numerical computations were performed with the finite element method (FEM) method in COMSOL Multiphysics 5.5. The mesh near the electrode/electrolyte interface was refined to resolve the double layer and to capture the gradients that occur within this region. The model domain was discretized into approximately $10^{5}$ elements, as further mesh refinement yielded relatively no significant change in the maximum velocities.

\section{RESULTS AND DISCUSSION}

The fluid dynamics that are characterized by the movements of the suspended tracer particles in hydrogen peroxide solution 
are shown in Figure 5. The electrolyte solution contained 0.3 $\mathrm{M}$ hydrogen peroxide, at $\mathrm{pH} 5.8$ and 4.2. The field of view is limited to a maximum height of about $350 \mu \mathrm{m}$, which originates from the midpoint at the surface the electrodes. The particle movements were recorded in this region to ascertain the directionality of the flow such that the resulting particle trajectories are considered to be the fluid flow streamlines (Figure 5a). Under bulk pH 5.8 condition, the particles are observed to be transported from $\mathrm{Pt}$ to $\mathrm{Au}$ (see Movie S1), and the displacement vector confirms the nature of the fluid flow to be electroosmotic that moves in the direction of the cathode from the anode (Figure $5 b$ ). Gold in this case serves as the cathode based on its higher mixed potential, as shown in the Tafel curve (Figure S1). The magnitude of the particle velocity is on the order of $10^{-6} \mathrm{~m} / \mathrm{s}$. At bulk $\mathrm{pH} 4.2$ the pumping direction is completely inverted and particles are advected from $\mathrm{Au}$ to $\mathrm{Pt}$, which indicates a completely reversed flow (Figure 5c, Movie S2). The particle velocity in this case is an order of magnitude lower than the case of $\mathrm{pH} 5.8$. In both cases, the movements of the charged particles appear to be slightly retarded around $y \leq 15 \mu \mathrm{m}$, due to the electrophoretic force that arises from interactions between the particle's surface charge and the induced electric field around this region (Figure S2). The $\zeta$ potential of the negative tracer particles was estimated to be $-34 \mathrm{mV}$ at $\mathrm{pH} 5.8$ and $-14 \mathrm{mV}$ at $\mathrm{pH} 4.2$ based on electrophoretic measurements conducted in our previous study. ${ }^{19}$ The electrophoretic force becomes stronger than the effective liquid drag force near the surface of the electrodes and makes the extraction of the fluid velocity more challenging at those locations.

The fluid flow features that are obtained from both bulk $\mathrm{pH}$ conditions indicate different reaction regimes that result in diverse flow behaviors. The reaction regimes, which we characterized by the dimensionless anodic Damköhler number $D a_{a}$, controls the generation of proton that influences the proton gradient. The proton gradient plays an important role in fluid flow generation via the self-induced electric field as will be shown in subsequent sections.

4.1. Fluorescence Lifetime Imaging. In this section, we present fluorescent lifetime results that characterize the reaction-induced proton concentration by FLIM. To prevent the disturbance of the fluorescence lifetime signal, the concentration of $\mathrm{H}_{2} \mathrm{O}_{2}$ was kept very dilute $\left(2 \mathrm{~mol} / \mathrm{m}^{3}\right)$, which minimizes the growth rate of the oxygen bubbles during the reaction. We first evaluated the performance of the bielectrode using the FLIM technique, by performing a blank test in the absence of hydrogen peroxide with a LysoSensor and water solution (Figure 6a). The spatial distribution of the fluorescence lifetime across the bielectrode region in the absence of $\mathrm{H}_{2} \mathrm{O}_{2}$ appears to be nearly homogeneous as the average lifetime for both electrodes was $\approx 2$, which implies no proton asymmetry across the electrodes. Figure $6 \mathrm{~b}$ shows the spatial variation of the fluorescence lifetime obtained across the bielectrode during the electrochemical reaction involving $\mathrm{H}_{2} \mathrm{O}_{2}$ $\left(2 \mathrm{~mol} / \mathrm{m}^{3}\right)$ at $\mathrm{pH}$ 5.8. An increase in the fluorescence lifetime signal is seen near the $\mathrm{Pt}$ region by a unit that indicates the enrichment of protons $\left(\mathrm{H}^{+}\right)$. In this case, the LysoSensor probe within the acidified regions becomes protonated, resulting in an increased lifetime signal. On the other hand, we observed a lower fluorescence lifetime signal near the $\mathrm{Au}$ region that indicates a reduction of proton concentration. In general, the average lifetime data demonstrates the establishment of a steady-state proton concentration gradient across the bielectrode, which is necessary for electrocatalytic actuation. We explored the nature of proton distribution associated with a lower anodic reactive regime by changing the $\mathrm{pH}$ of the solution to 4 , which corresponds to $\mathrm{Da}_{\mathrm{a}}=58$; the result is shown in Figure 6c.

It can be seen from Figure $6 \mathrm{c}$ that the fluorescence signal across the bielectrode is nearly homogeneous and the proton gradient appears to be diminished. The proton concentration is determined from the FLIM result and compared with simulation results that are plotted at different channel heights. The results match well at the cathode where protons are depleted and deviate at the anode. The simulation data suggests the establishment of a steeper gradient at the surface of the bielectrode. This highlights the limitation of FLIM toward resolving the near-surface region of the bielectrode (Figure 7). This fluorophores that are bounded to the reaction

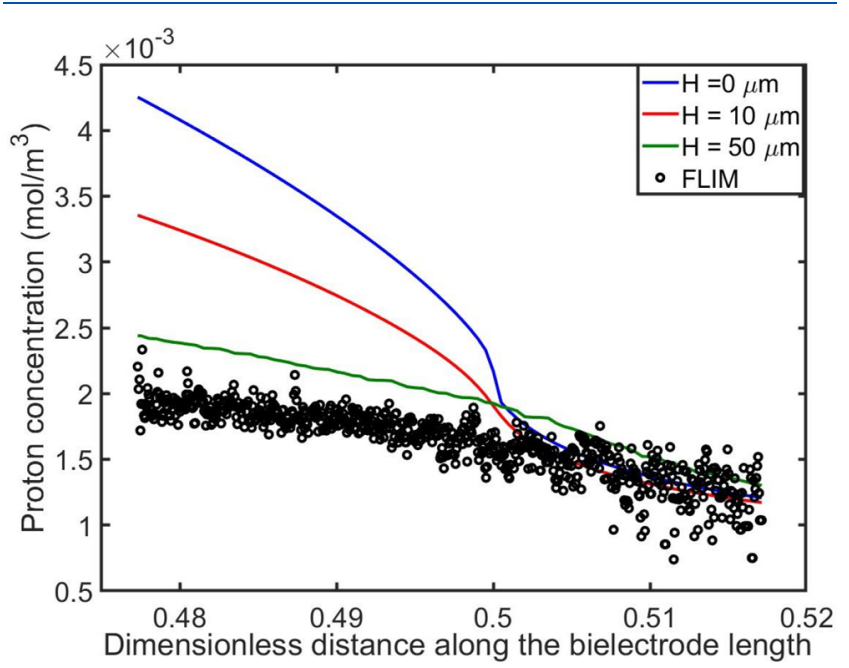

Figure 7. Proton concentration profile along the junction of the bielectrode for simulation (bulk $\mathrm{pH}$ 5.62) at channel heights and FLIM results. The solid lines represent the simulation results, and the open symbols depict the FLIM data. The distance was nondimensionalized by the length of the bielectrode, $L=10000 \mu \mathrm{m}$. The proton concentration was averaged at the electrode for FLIM.

plane at the near-surface region are overwhelmed by the background fluorescence coming from those in the bulk region. One way to circumvent this issue would be to couple total internal reflection fluorescence (TIRF) with FLIM, which can exclude the background fluorescence noise by selectively exciting the fluorophores situated at the near-surface region, and unravel the steep proton gradient that was predicted by the numerical model. ${ }^{38}$

4.2. Electrocatalytic Reaction Induced Flow Regimes. In this section, we characterized reaction regimes under bulk $\mathrm{pH}$ variations and studied their influence on the resulting flow dynamics numerically. The reaction regimes are defined by the reactivity ratio between the anodic and cathodic Damköhler numbers $\left(\mathrm{Da}_{\mathrm{a}} / \mathrm{Da}_{\mathrm{c}}\right)$ and varied to create regimes with different limiting mechanisms that control the overall electrocatalytic process. For each reactivity ratio, simulations were performed by varying the Damköhler numbers within the range $10^{\circ}-10^{3}$ that impacts the reaction kinetics. The maximum induced velocity is obtained at the junction of the bielectrode $\max (u(x=0.5, y=0))$ and plotted versus $\mathrm{Da}_{\mathrm{a}}$ numbers (Figure $8)$. It can be seen for every reactivity ratio in Figure 8 that the flow generated under bulk $\mathrm{pH} 4$ is completely reversed, while 
(a)

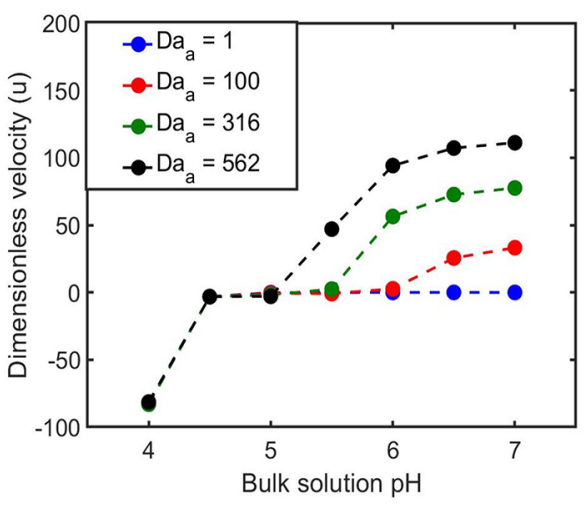

(c)

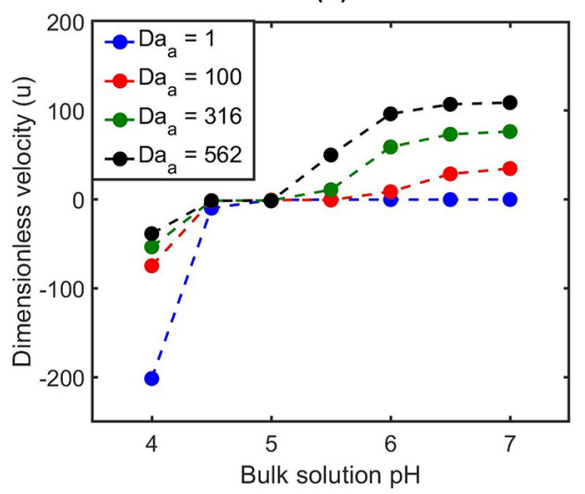

(b)
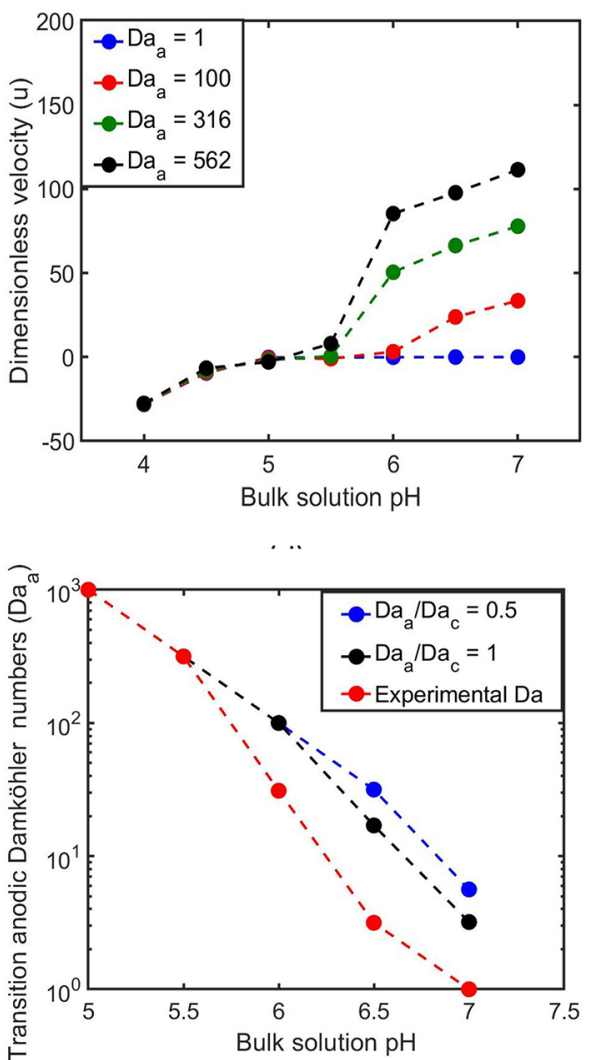

Figure 8. Simulation results for the maximum dimensionless velocity magnitude plotted versus $\mathrm{pH}$ for (a) reactivity ratio $\mathrm{Da}_{\mathrm{a}} / \mathrm{Da}_{\mathrm{c}}=0.5$, (b) reactivity ratio $\mathrm{Da}_{\mathrm{a}} / \mathrm{Da}_{\mathrm{c}}=1$, and (c) $\mathrm{Da}_{\mathrm{a}}=350$ and $\mathrm{Da}_{\mathrm{c}}=1$. (d) Transition $\mathrm{Da}_{\mathrm{a}}$ numbers that indicate the transition from reversed to normal flow and vice versa under different solution $\mathrm{pHs}$. The reactivity ratios are kept fixed, and the Damköhler numbers are varied in the range $10^{0}-10^{3}$. The experimental $\mathrm{Da}$ is defined as $\mathrm{Da}_{\mathrm{a}}=350$ and $\mathrm{Da}_{\mathrm{c}}=1$, and for this case only $\mathrm{Da}_{\mathrm{a}}$ is varied. The dotted-dashed lines are for the purpose of visualization only.

(a)

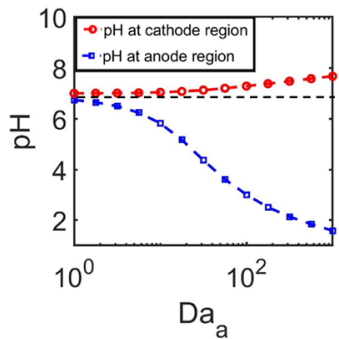

(b)

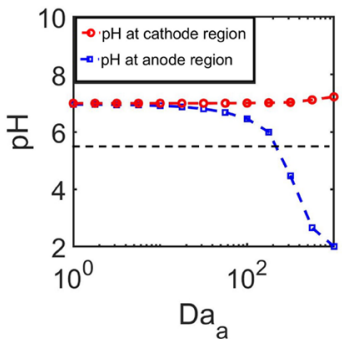

(c)

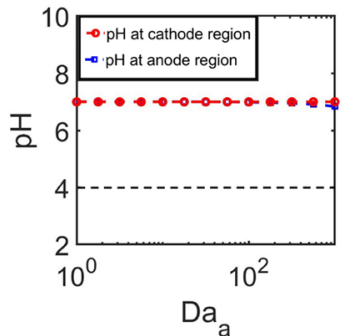

Figure 9. Average $\mathrm{pH}$ versus $\mathrm{Da}_{\mathrm{a}}$ evaluated at platinum and gold electrode surfaces for (a) $\mathrm{pH} 7$, (b) $\mathrm{pH} 5.5$, and (c) pH 4. The square symbols represent average $\mathrm{pH}$ at platinum, and the circular symbols denote average $\mathrm{pH}$ at gold. The black dashed line refers to the bulk $\mathrm{pH}$ of the electrolyte. The dashed lines are for the purpose of visualization only.

at $\mathrm{pH} 7$ the normal flow direction is obtained. Figure $8 \mathrm{~d}$ shows the $\mathrm{Da}_{\mathrm{a}}$ transition regimes under different bulk $\mathrm{pH}$ conditions below which the flow becomes fully reversed, and above which an electroosmotic flow is obtained. The transition points vary inversely with the electrolyte bulk $\mathrm{pH}$, which indicates the dominance of reactive time scales over diffusion, toward the reestablishment of normal flow under reaction regimes that are prone to flow reversal. This result highlights the interplay between the electrocatalytic and diffusion reactions toward the generation of surface induced flow, and the conservation of current under bulk $\mathrm{pH}$ variations.
We examine further the proton concentration gradient associated with the induced velocities for the case of $\mathrm{Da}_{\mathrm{a}} / \mathrm{Da}_{\mathrm{c}}=$ 1 at bulk $\mathrm{pH}$ values of $4,5.5$, and 7 . The variation of both Damköhler numbers within this fixed ratio represents the variation of the concentration of $\mathrm{H}_{2} \mathrm{O}_{2}$. The proton concentration here is defined by the average local $\mathrm{pH}$ at the electrode and bulk regions and presented in (Figure 9). For the case of bulk $\mathrm{pH} 7$, the proton concentration gradient builds up from the bulk $\mathrm{pH}$ and becomes more pronounced with increasing $\mathrm{Da}_{\mathrm{a}}$ (Figure 9a). In Figure $9 \mathrm{~b}$ the local proton concentration for bulk $\mathrm{pH} 5.5$ presents two distinct forms. At 
reaction regimes associated with flow reversal, $\mathrm{Da}_{\mathrm{a}} \leq 177$, the bulk $\mathrm{pH}$ profile falls outside the bounds of the $\mathrm{pH}$ profile for the anode and cathode due to the absence of charge asymmetry in the non-neutral zone, whereas from $\mathrm{Da}_{\mathrm{a}} \geq$ 316 , which is under the electroosmotic flow regime, the proton gradient offers a behavior similar to that for bulk $\mathrm{pH} 7$ and demonstrates sensitivity to the increased reaction rate. In Figure 9c, the proton gradient obtained for bulk $\mathrm{pH} 4$ becomes diminished and the flow under this regime is fully reversed.

Under steady-state conditions, the reactive current is constant. To achieve this, the system adjusts the cathodic reaction rate via the proton surface concentration through an induced potential, as the reaction rate at the anode is fixed by the concentration of hydrogen peroxide. ${ }^{37}$ The induced potentials at different reaction regimes under different bulk $\mathrm{pHs}$ for the case of $\mathrm{Da}_{\mathrm{a}} / \mathrm{Da}_{\mathrm{c}}=1$ are presented in Figure 10 .

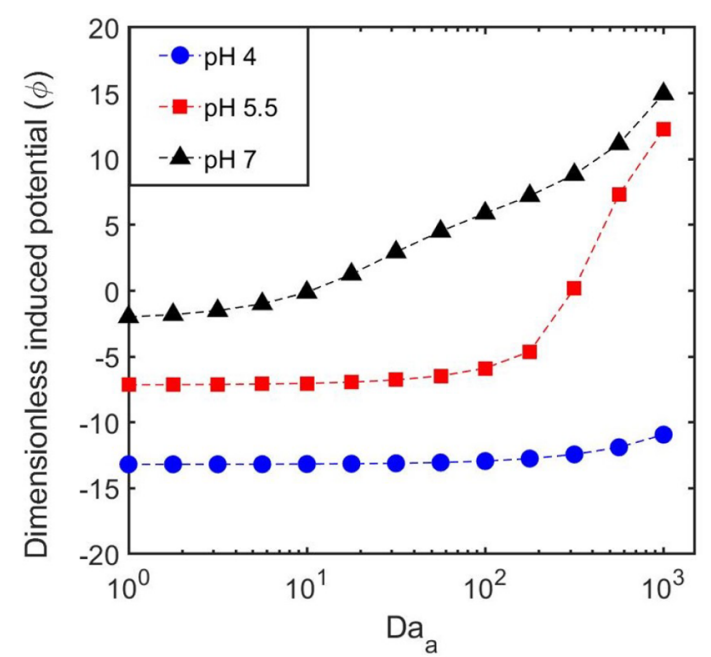

Figure 10. Effects of $\mathrm{Da}_{\mathrm{a}}$ on induced potential. The dotted-dashed lines are for the purpose of visualization only.
The potential induced for bulk $\mathrm{pH} 4$ is completely negative, and it corresponds to the completely reversed flow that is generated for the reactive regimes under this bulk $\mathrm{pH}$. Due to the excess of protons that are available by the bulk $\mathrm{pH}$, the induced potential for this case shifts more negative to enforce proton depletion via the reduction reaction as indicated by the negative potential. For the case of bulk $\mathrm{pH} \mathrm{7,} \mathrm{the} \mathrm{induced}$ potential for bulk $\mathrm{pH} 7$ is mostly positive except at $\mathrm{Da}_{\mathrm{a}} \leq 10$, where the oxidation reaction rate is completely lowered and the space charge density at the electrode is negative. For bulk $\mathrm{pH} 5.5$, the induced potential flips from negative to positive at $\mathrm{Da}_{\mathrm{a}}=316$, which is associated with the transition from reversed to normal flow (see Figure 9b).

We observed some interesting features regarding the electric field due to the proton gradient dynamics. Typically, the electric field is induced by the current of protons that originates from the anode toward the cathode. There exists an apparent source of electric field that results from the superposition of the electric field contributions from the proton flux and the surface charges. ${ }^{32,37,39}$ The location of the apparent source of the field lines can be influenced by the dynamics of the proton gradient under reaction regimes. In Figure 11 the electric field lines and flow field are presented for reaction regimes under bulk $\mathrm{pH}$ 5.5. For the case of $\mathrm{Da}_{\mathrm{a}}=3.1$ where flow is fully reversed, the apparent source of the field lines is situated above $\mathrm{Au}$ (Figure 11a). In Figure 11b the flow field and electric field lines are presented for the transition $\mathrm{Da}_{\mathrm{a}}$ reaction regime under bulk $\mathrm{pH} 5.5$, where the flow is fully reversed at $\mathrm{Da}_{\mathrm{a}}<316$ and the flow is driven from the anode to the cathode at $\mathrm{Da}_{\mathrm{a}}>316$. The onset and transition to either of the two aforementioned flow forms are characterized by the generation of a pair of counter-rotating vortices. The apparent source of the electric field lines for this case is doubled and situated above both electrodes. In Figure $11 \mathrm{c}$ at $\mathrm{Da}_{\mathrm{a}}=1000$, the apparent source of electric field lines is situated above Pt and points the cathode in accordance with the local flux of protons. For this case an electroosmotic flow is generated.

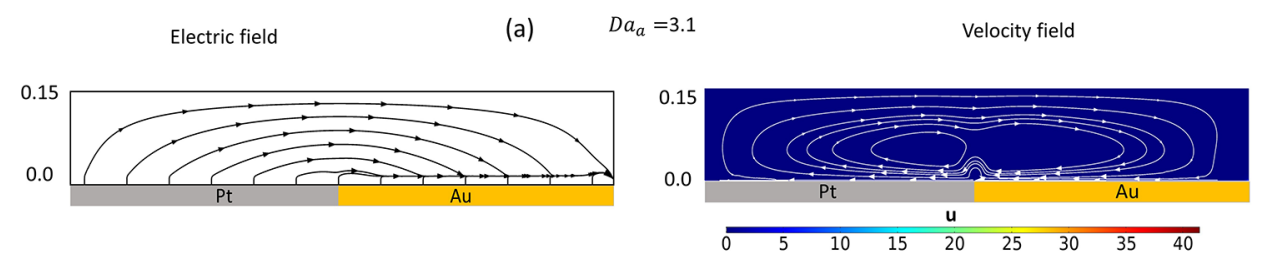

(b) $\quad D a_{a}=316$
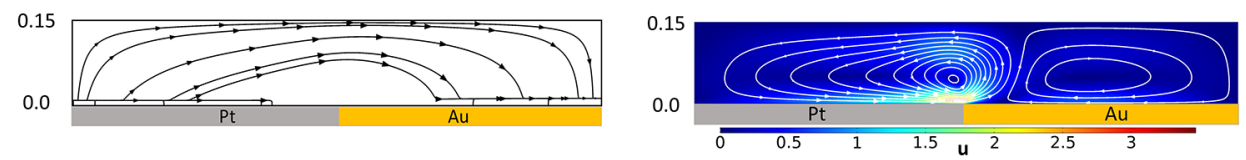

(c) $\quad D a_{a}=1000$
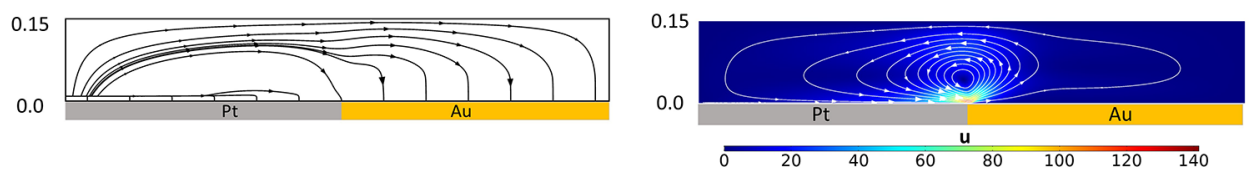

Figure 11. Simulation results for the electric field profile (left) and flow field (right) under reaction regimes (a) Daa $=3.1,(b) D a_{a}=316$, and (c) $\mathrm{Da}_{\mathrm{a}}=1000$. Black streamlines represent the electric field, and white streamlines represent the velocity fluid flow. The colorbar represents dimensionless velocity magnitude. The heights and widths are normalized with the bielectrode's length, $L=10 \mathrm{~mm}$. 


\section{CONCLUSION}

Electrocatalytic reaction driven flow has been studied for different anodic reaction regimes $\left(\mathrm{Da}_{\mathrm{a}}\right)$ via concentration and bulk $\mathrm{pH}$ variations, using an experimental and numerical analysis that highlights its dependence on the proton concentration gradient. The fluid flow is visualized using particle tracking, while the reaction-induced proton gradient is spatially mapped across the bielectrode with fluorescence lifetime imaging microscopy. Our numerical simulations show that the reversed flow is primarily influenced by the dynamics of the proton concentration gradient that strongly impacts the electric field, in conjunction with charge density imbalances within the non-neutral zones. The transition reaction regime at which the flow is transformed from its reversed to a normal form was presented. It highlights the manner in which the electrocatalytic system enforces a steady-state condition through the interplay between diffusive and reaction time scales via the induced potential that adjusts the surface concentration of $\mathrm{H}^{+}$ions, which affects the resulting advective flow and controls the overall electrocatalytic process. The proton gradient which is the basis for chemomechanical actuation was investigated using fluorescence lifetime imaging, which highlights an asymmetry of protons established across the electrodes during electrocatlytic reactions. The proton asymmetry is impacted by the reaction regimes under bulk $\mathrm{pH}$ conditions, where flow reversal occurs at $\mathrm{pH} \leq 4.5$. These results are relevant and crucial toward the exploitation of ERDF for potential applications such as micromixing.

\section{ASSOCIATED CONTENT}

\section{SI Supporting Information}

The Supporting Information is available free of charge at https://pubs.acs.org/doi/10.1021/acs.jpcc.1c06458.

Tafel plots presenting electrochemical characterization of $\mathrm{Pt}$ and $\mathrm{Au}$ electrodes; balance of forces acting on tracer particles as a function of height from the electrode within hydrogen peroxide at bulk pH 5.8 and 4.2 (PDF) Particle motion that highlights fluid flow generated at bulk pH 5.8 (MP4)

Particle motion that highlights fluid flow generated at bulk pH 4.2 (MP4)

\section{AUTHOR INFORMATION}

\section{Corresponding Author}

Rob G. H. Lammertink - Soft Matter, Fluidics and Interfaces, MESA+ Institute for Nanotechnology, University of Twente, 7522NB Enschede, The Netherlands; O orcid.org/00000002-0827-2946; Email: r.g.h.lammertink@utwente.nl

\section{Authors}

Abimbola A. Ashaju - Soft Matter, Fluidics and Interfaces, MESA+ Institute for Nanotechnology, University of Twente, 7522NB Enschede, The Netherlands

Veerle Otten - Soft Matter, Fluidics and Interfaces, MESA+ Institute for Nanotechnology, University of Twente, 7522NB Enschede, The Netherlands

Jeffery A. Wood - Soft Matter, Fluidics and Interfaces, MESA + Institute for Nanotechnology, University of Twente, 7522NB Enschede, The Netherlands; O orcid.org/00000002-9438-1048

Complete contact information is available at: https://pubs.acs.org/10.1021/acs.jpcc.1c06458

\section{Notes}

The authors declare no competing financial interest.

\section{ACKNOWLEDGMENTS}

This work is part of the research program "Stirring the boundary layer" with Project No. STW 016.160.312, which is financed by the Dutch Research Council (NWO). The authors thank Jan van Nieuwkasteele for technical assistance with the fabrication of the electrodes.

\section{REFERENCES}

(1) Soong, R. K.; Bachand, G. D.; Neves, H. P.; Olkhovets, A. G.; Craighead, H. G.; Montemagno, C. D. Powering an Inorganic Nanodevice with a Biomolecular Motor. Science 2000, 290, 15551558.

(2) Pantaloni, D.; Clainche, C. L.; Carlier, M.-F. Mechanism of Actin-Based Motility. Science 2001, 292, 1502-1506.

(3) Weibel, D. B.; Garstecki, P.; Ryan, D.; DiLuzio, W. R.; Mayer, M.; Seto, J. E.; Whitesides, G. M. Microoxen: Microorganisms to move microscale loads. Proc. Natl. Acad. Sci. U. S. A. 2005, 102, 11963-11967.

(4) Paxton, W. F.; Kistler, K. C.; Olmeda, C. C.; Sen, A.; St. Angelo, S. K.; Cao, Y.; Mallouk, T. E.; Lammert, P. E.; Crespi, V. H. Catalytic nanomotors: Autonomous movement of striped nanorods. J. Am. Chem. Soc. 2004, 126, 13424-13431.

(5) Wang, W.; Duan, W.; Zhang, Z.; Sun, M.; Sen, A.; Mallouk, T. E. A tale of two forces: Simultaneous chemical and acoustic propulsion of bimetallic micromotors. Chem. Commun. 2015, 51, 1020-1023.

(6) Paxton, W. F.; Baker, P. T.; Kline, T. R.; Wang, Y.; Mallouk, T. E.; Sen, A. Catalytically induced electrokinetics for motors and micropumps. J. Am. Chem. Soc. 2006, 128, 14881-14888.

(7) Kline, T. R.; Paxton, W. F.; Wang, Y.; Velegol, D.; Mallouk, T. E.; Sen, A. Catalytic Micropumps: Microscopic Convective Fluid Flow and Pattern Formation. J. Am. Chem. Soc. 2005, 127, 1715017151.

(8) Ibele, M. E.; Wang, Y.; Kline, T. R.; Mallouk, T. E.; Sen, A. Hydrazine fuels for bimetallic catalytic microfluidic pumping. J. Am. Chem. Soc. 2007, 129, 7762-7763.

(9) Serra-Maia, R.; Bellier, M.; Chastka, S.; Tranhuu, K.; Subowo, A.; Rimstidt, J. D.; Usov, P. M.; Morris, A. J.; Michel, F. M. Mechanism and Kinetics of Hydrogen Peroxide Decomposition on Platinum Nanocatalysts. ACS Appl. Mater. Interfaces 2018, 10, 21224-21234.

(10) Brown, A. T.; Poon, W. C. K.; Holm, C.; de Graaf, J. Ionic screening and dissociation are crucial for understanding chemical selfpropulsion in polar solvents. Soft Matter 2017, 13, 1200-1222.

(11) Brooks, A. M.; Tasinkevych, M.; Sabrina, S.; Velegol, D.; Sen, A.; Bishop, K. J. M. Shape-directed rotation of homogeneous micromotors via catalytic self-electrophoresis. Nat. Commun. 2019, 10, 495.

(12) Jun, I.-K.; Hess, H. A Biomimetic, Self-Pumping Membrane. Adv. Mater. 2010, 22, 4823-4825.

(13) Naeem, S.; Naeem, F.; Liu, J.; Quiñones, V. A. B.; Zhang, J.; He, L.; Huang, G.; Solovev, A. A.; Mei, Y. Oxygen Microbubble Generator Enabled by Tunable Catalytic Microtubes. Chem. - Asian J. 2019, 14, 2431-2434.

(14) Afshar Farniya, A.; Esplandiu, M. J.; Bachtold, A. Sequential Tasks Performed by Catalytic Pumps for Colloidal Crystallization. Langmuir 2014, 30, 11841-11845.

(15) Punckt, C.; Jan, L.; Jiang, P.; Frewen, T. A.; Saville, D. A.; Kevrekidis, I. G.; Aksay, I. A. Autonomous colloidal crystallization in a galvanic microreactor. J. Appl. Phys. 2012, 112, 074905.

(16) Wong, F.; Sen, A. Progress toward Light-Harvesting SelfElectrophoretic Motors: Highly Efficient Bimetallic Nanomotors and Micropumps in Halogen Media. ACS Nano 2016, 10, 7172-7179.

(17) Wang, Y.; Hernandez, R. M.; Bartlett, D. J.; Bingham, J. M.; Kline, T. R.; Sen, A.; Mallouk, T. E. Bipolar electrochemical 
mechanism for the propulsion of catalytic nanomotors in hydrogen peroxide solutions. Langmuir 2006, 22, 10451-10456.

(18) Farniya, A. A.; Esplandiu, M. J.; Reguera, D.; Bachtold, A. Imaging the proton concentration and mapping the spatial distribution of the electric field of catalytic micropumps. Phys. Rev. Lett. 2013, 111, 168301.

(19) Ashaju, A. A.; Wood, J. A.; Lammertink, R. G. H. Electrocatalytic reaction-driven flow. Phys. Rev. Fluids 2021, 6, 044004.

(20) Subramanian, S.; Catchmark, J. M. Control of Catalytically Generated Electroosmotic Fluid Flow through Surface Zeta Potential Engineering. J. Phys. Chem. C 2007, 111, 11959-11964.

(21) Leenheer, A. J.; Atwater, H. A. Imaging Water-Splitting Electrocatalysts with $\mathrm{pH}$-Sensing Confocal Fluorescence Microscopy. J. Electrochem. Soc. 2012, 159, H752-H757.

(22) Engstrom, R. C.; Ghaffari, S.; Qu, H. Fluorescence imaging of electrode-solution interfacial processes. Anal. Chem. 1992, 64, 25252529.

(23) Doneux, T.; Bouffier, L.; Goudeau, B.; Arbault, S. Coupling Electrochemistry with Fluorescence Confocal Microscopy To Investigate Electrochemical Reactivity: A Case Study with the Resazurin-Resorufin Fluorogenic Couple. Anal. Chem. 2016, 88, 6292-6300.

(24) Bowyer, W. J.; Xie, J.; Engstrom, R. C. Fluorescence Imaging of the Heterogeneous Reduction of Oxygen. Anal. Chem. 1996, 68, 2005-2009.

(25) de Valença, J.; Jõgi, M.; Wagterveld, R. M.; Karatay, E.; Wood, J. A.; Lammertink, R. G. H. Confined Electroconvective Vortices at Structured Ion Exchange Membranes. Langmuir 2018, 34, 24552463.

(26) Benneker, A. M.; Wood, J. A.; Tsai, P. A.; Lammertink, R. G. H. Observation and experimental investigation of confinement effects on ion transport and electrokinetic flows at the microscale. Sci. Rep. 2016, 6, 37236.

(27) Principles of Fluorescence Spectroscopy; Lakowicz, J. R., Ed.; Springer US: 2006.

(28) Datta, R.; Heaster, T. M.; Sharick, J. T.; Gillette, A. A.; Skala, M. C. Fluorescence lifetime imaging microscopy: fundamentals and advances in instrumentation, analysis, and applications. J. Biomed. Opt. 2020, 25, 1-43.

(29) Thielicke, W.; Stamhuis, E. J. PIVlab - Towards User-friendly, Affordable and Accurate Digital Particle Image Velocimetry in MATLAB. J. Open Res. Softw. 2014, 2, e30.

(30) Lin, H.-J.; Herman, P.; Kang, J. S.; Lakowicz, J. R. Fluorescence Lifetime Characterization of Novel Low-pH Probes. Anal. Biochem. 2001, 294, 118-125.

(31) Johnson, I. Molecular Probes Handbook: A Guide to Fluorescent Probes and Labeling Technologies; Life Technologies Corp.: 2010.

(32) Moran, J. L.; Posner, J. D. Electrokinetic locomotion due to reaction-induced charge auto-electrophoresis. J. Fluid Mech. 2011, 680, 31-66.

(33) Sabass, B.; Seifert, U. Nonlinear, electrocatalytic swimming in the presence of salt. J. Chem. Phys. 2012, 136, 214507.

(34) Bazant, M. Z.; Chu, K. T.; Bayly, B. J. Current-voltage relations for electrochemical thin films. SIAM J. Appl. Math. 2005, 65, 14631484.

(35) Biesheuvel, P.; van Soestbergen, M.; Bazant, M. Imposed currents in galvanic cells. Electrochim. Acta 2009, 54, 4857-4871.

(36) Delahay, P. Double layer and electrode kinetics; Interscience Publishers, 1965.

(37) Davidson, S. M.; Lammertink, R. G.; Mani, A. Predictive model for convective flows induced by surface reactivity contrast. Phys. Rev. Fluids 2018, 3, 053701.

(38) Fish, K. N. Total Internal Reflection Fluorescence (TIRF) Microscopy. Curr. Protoc. Cytom. 2009, 50, 12.18.1-12.18.13.

(39) Esplandiu, M. J.; Afshar Farniya, A.; Reguera, D. Key parameters controlling the performance of catalytic motors. J. Chem. Phys. 2016, 144, 124702. 